Nelson Alexander Pérez García

Modelamento de Efeitos de Atenuação por Chuvas em Enlaces Terrestres Ponto-a-ponto e Ponto-multiponto

Tese de Doutorado

Tese apresentada como requisito parcial para obtenção do título de Doutor pelo Programa de PósGraduação em Engenharia Elétrica da PUC-Rio.

Orientador: Prof. Luiz Alencar Reis da Silva Mello

Rio de Janeiro, RJ, 13 de junho de 2003 
Nelson Alexander Pérez García

\section{Modelamento de Efeitos de Atenuação por Chuvas em Enlaces Terrestres Ponto-a-ponto e Ponto-multiponto}

Tese apresentada como requisito parcial para obtenção do título de Doutor pelo Programa de Pós-Graduação em Engenharia Elétrica da PUC-Rio. Aprovada pela Comissão Examinadora abaixo assinada.

Prof. Luiz Alencar Reis da Silva Mello Orientador Centro de Estudos em Telecomunicações - CETUC-PUCIRio

Dr. Luís Cláudio Palma Pereira CPqD

Dr. Eduardo Javier Arancibia Vásquez TIM-Brasil

Prof. Mauro Soares de Assis Instituto Militar de Engenharia - IME

Dr. Erasmus Couto Brazil de Miranda Centro de Estudos em Telecomunicações

Dr. Gláucio Lima Siqueira Centro de Estudos em Telecomunicações - CETUC-PUCIRio

Prof. Ney Augusto Dumont Coordenador(a) Setorial do Centro Técnico Científico - PUC-Rio 
Todos os direitos reservados. É proibida a reprodução total ou parcial do trabalho sem autorização da universidade, do autor e do orientador.

\section{Nelson Alexander Pérez García}

Gradou-se em Engenharia Elétrica na ULA (Universidad de Los Andes), Mérida, Venezuela, em 1992. Em 2000 recebeu o título de mestre em Engenharia Elétrica, na área de Electromagnetismo Aplicado, no CETUC-PUC $\backslash$ Rio. Desde 1994 é Profesor em Telecomunicações na ULA. Sua área de pesquisa está orientada ao desenvolvimento de modelos para caracterização dos efeitos de propagação que influem no planejamento e dimensionamento de sistemas sem fio de comunicaciones móveis e radio acesso.

Ficha Catalográfica

Pérez García, Nelson Alexander

Modelamento de Efeitos de Atenuação por Chuvas em Enlaces Terrestres Ponto-a-ponto e Ponto-multiponto / Nelson Alexander Pérez García; orientador: Luiz Alencar Reis da Silva Mello. - Rio de Janeiro: PUC, Departamento de Engenharia Elétrica, 2003

175 f: il. ; $30 \mathrm{~cm}$

Tese (doutorado) - Pontifícia Universidade Católica do Rio de Janeiro, Departamento de Engenharia Elétrica

1. Engenharia elétrica - Teses. 2. Sistemas sem fio. 3. Atenuação por chuvas. 4. Modelamento. 5. Planejamento. I. Mello, Luiz Alencar Reis da Silva. II. Pontifícia Universidade Católica do Rio de Janeiro, Departamento de Engenharia Elétrica. III. Título 
A Deus Todo Poderoso, a minha mamãe María, a minha esposa Carmelina e a meus filhos Tawny e Bruno. Também para ti, minha eterna pequenina María Auxiliadora (in memoriam) 


\section{Agradecimentos}

A meu orientador, Prof. Luiz A. R. da Silva Mello, pela sua contínua assessoria e apoio pessoal, moral e profissional. Obrigado pela confiança em mim depositada.

À Prof. Marlene Sabino Pontes, quem facilitou minha adaptação inicial ao Brasil e sempre me deu incentivo profissional.

Ao Prof. Erasmus Couto de Miranda, por suas importantes orientações que ajudaram ao desenvolvimento desta tese.

Ao conjunto de amigos venezuelanos residentes no Rio de Janeiro, especialmente aos do grupo de Softball, presentes em todo momento.

Aos meus amigos colombianos Sergio e Maria Cristina, que sempre estiveram presentes nos momentos de dificuldade.

A meus amigos e colegas brasileiros Marta e Luis Carlos Linares, dos quais sempre recebi palavras de ânimo e estímulo para continuar adiante.

Ao pessoal do CETUC em geral, cuja gentileza me fizeram sentir como em casa.

Ao CNPq, pelo importante apoio econômico recebido durante este trabalho.

A minha família e amigos na Venezuela, permanente fonte de inspiração.

Á Fundación Nacional de Investigaciones Científicas y Tecnológicas, FONACIT (Caracas, Venezuela), quem fez possível minha permanência no Brasil, durante este período.

À Universidad de Los Andes, ULA (Mérida, Venezuela), instituição em cuja representação me desenvolvo e que também fez possível minha permanência aqui no Brasil, para o cumprimento deste importante objetivo. 


\section{Resumo}

Pérez García, Nelson Alexander. Modelamento de Efeitos de Atenuação por Chuvas em Enlaces Terrestres Ponto-a-ponto e Ponto-multiponto. Rio de Janeiro, RJ, 2003. 175p. Tese de Doutorado - Departamento de Engenharia Elétrica, Pontifícia Universidade Católica do Rio de Janeiro.

A crescente demanda dos usuários por serviços de banda larga tais como dados a alta velocidade, vídeo, aúdio e multimídia, tem levado ao uso de sistemas rádio ponto-multiponto em freqüências superiores a $10 \mathrm{GHz}$, que além de menos utilizadas, permitem oferecer as grandes larguras de banda necessárias. Nessas freqüências, as perdas de propagação do sinal devem-se principalmente ao efeito de chuvas. A atenuação por chuvas afeta diretamente a cobertura e o desempenho dos sistemas que operam nestas faixas de freqüência. No presente trabalho é desenvolvido um modelo empírico para a previsão da distribuição cumulativa da atenuação por chuvas, utilizando a base oficial de ITU-R e resultados experimentais de medidas realizadas no Brasil. Este modelo fornece resultados mais precisos do que os atualmente propostos na literatura técnica e permite avaliar o impacto das chuvas na cobertura de sistemas ponto-multiponto tipo LMDS (Local Multipoint Distribution System). Além disto, foram desenvolvidos dois modelos para a previsão da distribuição da atenuação diferencial por chuvas, fator essencial para a determinação de efeitops de interferência e seu impacto das chuvas no desempenho de sistemas LMDS. Finalmente, foram desenvolvidos modelos para a previsão da duração total e média, de eventos de atenuação por chuvas, estatísticas também relevantes na análise de performance de sistemas LMDS.

\section{Palavras-chave}

Sistemas sem fio, Atenuação por chuvas, Modelamento, Planejamento 


\section{Resumo}

Pérez García, Nelson Alexander. Modelling to Rain Attenuation Efects in Point-to-point and Point-to-multipoint Terrestrial Links. Rio de Janeiro, RJ, 2003. 175p. D.Sc. These - Departamento de Engenharia Elétrica, Pontifícia Universidade Católica do Rio de Janeiro.

The growing demand for broadband services such as high-speed data, video, audio and multimedia, has lead to the use of point-multipoint radio systems operating in frequencies above of $10 \mathrm{GHz}$, that are not yet allocated for other services and allow the use of the large bandwidths required by these services. At these frequencies, the main contribution for the propagation losses is due to rain attenuation. The rain directly affects the area coverage and the performance of systems operating at these frequencies. In the present work, an empirical model is developed for predicting the cumulative distribution of rain attenuation prediction, using the ITU-R data base and results of propagation measurements campaigns performed in Brazil. The model provides better results than others currently found in the technical literature and allows the evaluation of the impact of rain attenuation in the coverage of LMDS (Local Multipoint Distribution System/Local Multipoint Communications System). Also, two models were developed for predicting distributions of differential rain attenuation, an essential factor for interference effects and its impact in LMDS performance. Finally, models were developed for the prediction of total and average duration of rain attenuation events, also relevant for systems performance assessment.

\section{Keywords}

Wireless systems, rain attenuation, modelling, planning 


\section{Sumário}

1 INTRODUÇÃO 20

1.1. DESCRIÇÃO DO PROBLEMA 20

1.2. OBJETIVOS DO TRABALHO 25

1.3. RESULTADOS OBTIDOS E CONTRIBUIÇÕES DESTE TRABALHO 26

1.4. ORGANIZACÃO DO TEXTO 27

2 EFEITOS E MODELOS DA ATENUAÇÃO POR CHUVAS 29

2.1. ABSORÇÃO E ESPALHAMENTO DAS GOTAS DE ÁGUA 30

2.2. FATORES QUE INFLUEM NA ATENUAÇÃO POR CHUVAS 31

2.3. MODELOS DE PREVISÃO DA ATENUAÇÃO POR CHUVAS 33

2.3.1. Cálculo da Atenuação Específica 33

2.3.2. Modelo de ITU-R (Recomendação P.530-10) 36

2.3.3. Modelo de Crane 39

2.3.4. Modelo Australiano (Documento 3M/38-E, de ITU-R) 41

2.3.5. Modelo CETUC-98 41

2.4. DISTRIBUIÇÕES DA TAXA DE PRECIPITAÇÃO 43

2.5. ATENUAÇÃO DIFERENCIAL POR CHUVAS 44

2.6. DURAÇÃO E NÚMERO DE EVENTOS DE CHUVA

2.7. DISTRIBUIÇÃO DE WEIBULL 47

2.7.1. Distribuição de Weibull de Três Parâmetros 47

2.7.2. Distribuição de Weibull de Dois Parâmetros 48

3 RESULTADOS EXPERIMENTAIS $\quad 49$

3.1. BASE DE DADOS DE ITU-R 49

3.2. EXPERIMENTOS REALIZADOS NO BRASIL 50

3.2.1. Descrição do Experimento 53

3.2.2. Pré-processamento de Séries Temporais 55

3.3. ANÁLISE DE DADOS 56

3.3.1. Distribuição Cumulativa da Taxa de Precipitação 57 
3.3.2. Distribuição Cumulativa da Atenuação por Chuvas

3.3.3. Distribuição Cumulativa da Atenuação Diferencial

3.3.4. Distribuição Cumulativa da Duração e Número de Eventos de Chuva 59

3.3.5. Resultados

60

4 PREVISÃO E MODELAMENTO DA ATENUAÇÃO POR CHUVAS EM FREQÜÊNCIAS SUPERIORES A $10 \mathrm{GHZ}$

69

4.1. TESTES DE MODELOS EXISTENTES PARA A PREVISÃO DA ATENUAÇÃO POR CHUVAS 69

4.1.1. Resultados dos Testes 69

4.2. MODELAMENTO DA ATENUAÇÃO POR CHUVAS 77

4.2.1. Determinação da Expressão Empírica para a Previsão da Atenuação por Chuvas $\quad 79$

4.2.2. Comparação com as Medidas $\quad 84$

4.2.3. Teste de Validade 88

4.2.4. Atenuação por Chuvas no Brasil: Um Caso Particular 93

4.3. MODELAMENTO DA ATENUAÇÃO DIFERENCIAL POR CHUVAS PARA CLIMAS TROPICAIS E EQUATORIAIS 94

4.3.1. Modelo 1 para a Previsão da Atenuação Diferencial por Chuvas 95

4.3.2. Modelo 2 para a Previsão da Atenuação Diferencial por Chuvas 100

4.3.3. Comparação entre os Modelos 1 e 2

5 CARACTERÍSTICAS DINÂMICAS DA ATENUAÇÃO POR

CHUVAS

5.1. ANÁLISE DO COMPORTAMENTO ESTATÍSTICO DA

DURAÇÃO E NÚMERO DE EVENTOS DE CHUVA

5.2. MODELAMENTO DA DISTRIBUIÇÃO CUMULATIVA DA DURAÇÃO DE EVENTOS DE CHUVA PARA CLIMAS TROPICAIS E EQUATORIAIS

5.2.1. Determinação dos Parâmetros $\eta$ e $\beta$ da Distribuição da

Duração de Eventos 
5.2.3. Determinação da Expresão de $\beta$

5.2.4. Testes do Modelo DEC-PM 127

5.2.5. Modelo DEC-PM modificado 129

5.2.6. Comparação entre os Modelos DEC-PM e DEC-PM Modificado 132

5.3. MODELAMENTO DA DURAÇÃO MÉDIA DE EVENTOS

DE CHUVA 134

5.3.1. Coeficientes do Polinômio de Ajuste 135

5.3.2. Determinação das Funções para os Coeficientes do Polinômio de Ajuste 136

5.3.3. Teste do Modelo DMEC-PM 138

5.3.4. Modelo DMEC-PM Modificado 139

5.3.5. Comparação dos Modelos DMEC-PM e DMEC-PM Modificado 141

6 APLICAÇÃO DOS MODELOS DESENVOLVIDOS

6.1. SISTEMA LMDS 144

6.2. PREVISÃO DA COBERTURA EM SISTEMAS LMDS 145

6.2.1. Perdas de Propagação 145

6.2.2. Perda Máxima de Propagação 146

6.2.3. Determinação do Raio da Célula 147

6.2.4. Exemplo de Cálculo de Cobertura em Sistemas LMDS 147

6.3. DEGRADAÇÃO DA RELACÃO S/I DEVIDO À CHUVA 148

6.4. APLICAÇÃO DO MODELO DEC-PM Modificado 153

7 CONCLUSÕES E SUGESTÕES PARA TRABALHOS FUTUROS 155

7.1. CONCLUSÕES 155

7.2. SUGESTÕES PARA TRABALHOS FUTUROS 157

REFERÊNCIAS BIBLIOGRÁFICAS 159

APÊNDICE A BASE OFICIAL DE DADOS DE ITU-R (DBSG5) 164

APÊNDICE B CARACTERÍSTICAS DOS PARES DE ENLACES

CONVERGENTES DE SÃO PAULO E BRASILÍA 167 
APÊNDICE C BASE DE DADOS PARA O MODELAMENTO DA DURAÇÃO DE EVENTOS DE CHUVA NO BRASIL

APÊNDICE D DURAÇÃO MÉDIA DE EVENTOS DE CHUVA EM SÃO PAULO

APÊNDICE E RELAÇÃO ENTRE DURAÇÃO MÉDIA DE EVENTOS DE CHUVA E COMPRIMENTO DE ENLACE 


\section{Lista de figuras}

Figura 1 Técnica de polarização alternada no sistema LMDS. 22

Figura 2 Variação de raio de uma célula com a chuva, no sistema LMDS. 22

Figura 3 Atenuação por absorção. $\quad 30$

Figura 4 Atenuação por espalhamento. 31

Figura 5 Geometria para a determinação do fator de redução do modelo de ITU-R.

Figura 6 Distribuição cumulativa da atenuação por chuva no enlace Barueri-RIS.

Figura 7 Taxa de precipitação excedida durante 0,01\% do tempo. 44

Figura 8 Situação de atenuação diferencial por chuvas. 45

Figura 9 Serie temporal de atenuação por chuvas. 46

Figura 10 . Distribuição geográfica dos 7 enlaces convergentes de São Paulo. 51

Figura 11 Distribuição geográfica dos 6 enlaces convergentes de Brasília. $\quad 52$

Figura 12 Set-up experimental das medidas da atenuação por chuvas nos enlaces convergentes de São Paulo e Brasília. 53

Figura 13 Esquema básico da UAD. 54

Figura 14 Série temporal da potência recebida e da taxa de precipitação, gerada pelo programa TSEDIT. 55

Figura 15 Distribuições da taxa de precipitação nos enlaces convergentes de São Paulo. $\quad 62$

Figura 16 Distribuições cumulativas da atenuação por chuvas dos enlaces de São Paulo, para o primeiro ano de medidas (entre 0,01 e 1\%). 62

Figura 17 Distribuições cumulativas da atenuação total e diferencial para o enlace

$\begin{array}{ll}\text { Barueri-RIS. } & 64\end{array}$

Figura 18 Distribuições cumulativas da duração de eventos de chuvas para os 7 enlaces convergentes de São Paulo. $\quad 66$

Figura 19 Distribuições cumulativas do número de eventos de chuvas para os 7 enlaces convergentes de São Paulo. $\quad 67$

Figura 20 Distribuições cumulativas da atenuação total e diferencial para o enlace INCRA-BSA. 
Figura 21 Média dos erros (Recomendação P.311-10). 72

Figura 22 Desvio padrão dos erros (Recomendação P.311-10). 73

Figura 23 Valor RMS dos erros (Recomendação P.311-10). 73

Figura 24 Média dos erros (Erro relativo). 75

Figura 25 Desvio padrão dos erros (Erro relativo). $\quad 76$

Figura 26 Valor RMS dos erros (Erro relativo). 76

$\begin{array}{ll}\text { Figura } 27 \text { Gráfico de espalhamento de } \mathrm{r}_{\mathrm{p}} \text { vs. } \mathrm{R}_{\mathrm{p}} \text {. } & 80\end{array}$

Figura 28 Gráfico de espalhamento de $\mathrm{r}_{\mathrm{p}}$ vs. $\mathrm{d}$. 81

Figura 29 Gráfico de espalhamento de $r_{p}$ vs. Latitude. 81

Figura 30 Valores previstos versus valores medidos, do fator $r_{p}$. 83

Figura 31 Distribuição dos resíduos do fator $\mathrm{r}_{\mathrm{p}}$. 83

Figura 32 Média dos erros (Erro relativo), incluindo o modelo Pérez-Mello. 85

Figura 33 Desvio padrão dos erros (Erro relativo), incluindo o modelo PérezMello. $\quad 86$

Figura 34 Valor RMS dos erros (Erro relativo), incluindo o modelo Pérez-Mello.

Figura 35 Distribuições cumulativas, medidas e previstas, da atenuação por chuva, $\begin{array}{ll}\text { no enlace Chibolton. } & 87\end{array}$

Figura 36 Distribuições cumulativas, medidas e previstas, da atenuação por chuva, no enlace CENESP15. 88

Figura 37 Valor RMS dos erros (Erro relativo), para $\mathrm{d} \leq 5 \mathrm{~km} . \quad 89$

Figura 38 Valor RMS dos erros (Erro relativo) para $5 \mathrm{~km}<\mathrm{d} \leq 20 \mathrm{~km}$.

Figura 39 Valor RMS dos erros (Erro relativo), para d $>20 \mathrm{~km}$. 90

Figura 40 Valor RMS dos erros (Erro relativo), para $\mathrm{f} \leq 20 \mathrm{GHz}$.

Figura 41 Valor RMS dos erros (Erro relativo) para $20 \mathrm{GHz}<\mathrm{f} \leq 40 \mathrm{GHz} . \quad 91$

Figura 42 Valor RMS dos erros (Erro relativo) para f $>40 \mathrm{GHz} . \quad 92$

Figura 43 Valor RMS dos erros (Erro relativo) para latitudes $\leq 30^{\circ}$. 92

Figura 44 Valor RMS dos erros (Erro relativo) para latitudes $>30^{\circ}$.

Figura 45 Valor RMS dos erros (Erro Relativo), para as medidas no Brasil. 94

Figura 46 Gráfico de espalhamento de $\mathrm{A}_{\mathrm{ab}}$ vs. $\mathrm{A}_{\mathrm{a}}$. 95

Figura 47 Gráfico de espalhamento de $A_{a b}$ vs. $A_{b}$. 96

Figura 48 Gráfico de espalhamento de $\mathrm{A}_{\mathrm{ab}}$ vs. $|\Delta \mathrm{d}|$. 96

Figura 49 Gráfico de espalhamento de $\mathrm{A}_{\mathrm{ab}}$ vs. $\theta$. 
Figura 50 Valores observados versus previstos (modelo 1) da atenuação diferencial. $\quad 99$

Figura 51 Distribuição dos resíduos para o modelo 1.

Figura 52 Percentagens de tempo observadas vs previstas (enlace PGR-BNDES).

Figura 53 Percentagens de tempo observadas vs previstas (enlace BNDES-PGR).

Figura 54 Parâmetro $\eta$ vs. freqüência de operação de cada par de enlace. 102

Figura 55 Parâmetro $\eta$ vs. comprimento do enlace 1.

Figura 56 Parâmetro $\eta$ vs. comprimento do enlace 2.

Figura 57 Parâmetro $\eta$ vs. o ângulo entre os percursos dos enlaces. 104

Figura 58 Valores observados versus valores previstos, do parâmetro $\eta$. 105

Figura 59 Distribuição dos resíduos para $\eta$. 105

Figura 60 Parâmetro $\beta$ vs. freqüência de operação de cada par de enlace. 106

Figura 61 Parâmetro $\beta$ vs. comprimento do enlace 1. 106

Figura 62 Parâmetro $\beta$ vs. comprimento do enlace 2.

Figura 63 Parâmetro $\beta$ vs. o ângulo entre os percursos dos enlaces. 107

Figura 64 Valores observados vs. valores previstos para $\beta . \quad 108$

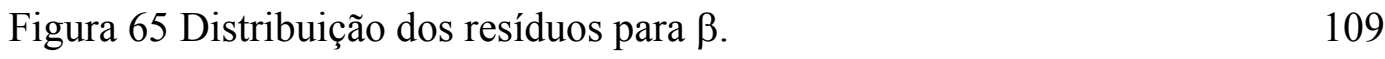

Figura 66 Valores observados vs. previstos (modelo 2) da atenuação diferencial.

Figura 67 Média dos erros (Erro relativo), para os Modelos 1 e 2.

Figura 68 Desvio padrão dos erros (Erro relativo), para os modelos 1 e $2 . \quad 112$

Figura 69 Valor RMS dos erros (Erro relativo), para os modelos 1 e 2. 112

Figura 70 Distribuições cumulativas, medidas e previstas, da atenuação diferencial por chuva, para o par Barueri-CENESP15. 114

Figura 71 Distribuições cumulativas, medidas e previstas, da atenuação diferencial por chuva, para o par PGR-INCRA.

Figura 72 Distribuições cumulativas medida e prevista da duração de eventos de atenuação por chuvas para o enlace CENESP15-RIS (limiar de $10 \mathrm{~dB}$ ). 117

Figura 73 Distribuições cumulativas medida e prevista do número de eventos de atenuação por chuvas para o enlace CENESP15-RIS (limiar de 10 dB). 117

Figura 74 Duração média de eventos de chuva, para o enlace CENESP15-RIS. 119 
Figura 75 Parâmetro $\eta$ versus o comprimento do enlace.

Figura 76 Parâmetro $\eta$ versus o nível de atenuação.

Figura 77 Parâmetro $\eta$ versus a duração máxima por nível de atenuação. 121

Figura 78 Valores de $\eta$ previstos versus valores de $\eta$ observados. 123

Figura 79 Distribuição dos resíduos para o parâmetro $\eta$. 123

$\begin{array}{ll}\text { Figura } 80 \text { Parâmetro } \beta \text { vs. comprimento do enlace. } & 124\end{array}$

$\begin{array}{ll}\text { Figura } 81 \text { Parâmetro } \beta \text { vs. nível de atenuação. } & 124\end{array}$

Figura 82 Parâmetro $\beta$ vs. duração máxima por nível de atenuação. 125

Figura 83 Valores de $\beta$ previstos versus valores de $\beta$ observados. $\quad 126$

Figura 84 Distribuição dos resíduos para o parâmetro $\beta$. 126

Figura 85 Média, desvio padrão e valor RMS dos erros (Recomendação P.31110), para o modelo DEC-PM.

Figura 86 Média, desvio padrão e valor RMS dos erros (Erro relativo), para o modelo DEC-PM.

128

Figura 87 Valores de $\eta$ previstos versus valores de $\eta$ observados (Modelo DECPM Modificado).

Figura 88 Distribuição dos resíduos para o parâmetro $\eta$ (Modelo DEC-PM Modificado).

Figura 89 Valores de $\beta$ previstos versus valores de $\beta$ observados (Modelo DECPM Modificado).

Figura 90 Distribuição dos resíduos para o parâmetro $\beta$ (Modelo DEC-PM Modificado).

Figura 91 Média dos erros para os modelos DEC-PM e DEC-PM modificado. 133

Figura 92 Desvio padrão dos erros para os modelos DEC-PM e DEC-PM modificado.

Figura 93 Valor RMS dos erros - modelos DEC-PM e DEC-PM modificado. 134

Figura 94 Distribuições cumulativas, medida e previstas, para o enlace Scania e um nível de atenuação de $15 \mathrm{~dB}$.

Figura 95 Valores observados da duração média de eventos de chuva versus valores

previstos da duração média de eventos de chuva.

Figura 96 Média, desvio padrão e valor RMS dos erros (Recomendação P.311- 
10), para o modelo DMEC-PM.

Figura 97 Média, desvio padrão e valor RMS dos erros (Erro Relativo), para o modelo DMEC-PM

Figura 98 Valores observados e previstos da duração média de eventos de atenuação por chuvas - modelo DMEC-PM Modificado.

Figura 99 Média dos erros (erro relativo), para os modelos DMEC-PM e DMECPM modificado.

Figura 100 Desvio padrão dos erros (erro relativo), para os Modelos DMEC-PM e DMEC-PM Modificado.

Figura 101 Valor RMS dos erros (erro elativo), para os Modelos DMEC-PM e DMEC-PM Modificado.

Figura 102 Fator de reuso numa célula.

Figura 103 Fator de reuso numa célula aplicando a polarização alternada. 149

Figura 104 Cenário de interferência no sistema LMDS.

Figura 105 Valores da relação S/I no sistema LMDS $\backslash$ LMCS, sob condições de chuva.

Figura 106 Curva de espalhamento entre a duração máxima de eventos por nível versus o nível de atenuação.

170

Figura 107 Duração média de eventos de chuva (Barueri-RIS).

Figura 108 Duração média de eventos de chuva (Bradesco2-RIS).

Figura 109 Duração média de eventos de chuva (CENESP15-RIS).

Figura 110 Duração média de eventos de chuva (CENESP18-RIS).

Figura 111 Duração média de eventos de chuva (Scania-RIS).

Figura 112 Duração média de eventos de chuva (Paranapiacaba-RIS). 174

Figura 113 Duração média de eventos de chuva (Shell-RIS).

Figura 114 Curva de espalhamento entre a duração máxima de eventos por enlace versus o comprimento do enlace. 


\section{Lista de tabelas}

Tabela 1 Características dos Sistemas BWS. 21

Tabela 2 Coeficientes para cálculo da atenuação específica. 35

Tabela 3 Regiões climáticas de ITU-R. 43

Tabela 4 Freqüências de operação dos enlaces da base de dados DB-SG5. 49

Tabela 5 Comprimentos dos enlaces da base de dados DB-SG5. 50

Tabela 6 Dados dos enlaces terrestres convergentes de São Paulo. 51

Tabela 7 Dados dos enlaces terrestres convergentes de Brasília. 52

Tabela 8 Distribuições cumulativas da taxa de precipitação para os 7 enlaces $\begin{array}{ll}\text { convergentes de São Paulo. } & 61\end{array}$

Tabela 9 Distribuição cumulativa da atenuação por chuvas para os 7 enlaces convergentes de São Paulo (entre 0,001 e 0,1\%). 61

Tabela 10 Distribuição cumulativa da atenuação diferencial por chuvas para os pares de enlaces convergentes de São Paulo que incluem Barueri-RIS. 63

Tabela 11 Distribuições cumulativas da duração de eventos de chuvas para o enlace CENESP15-RIS. $\quad 65$

Tabela 12 Distribuições cumulativas do número de eventos de chuvas para o enlace CENESP15-RIS.

Tabela 13 Distribuição cumulativa da taxa de precipitação para os 6 enlaces convergentes de Brasília. $\quad 67$

Tabela 14 Distribuição cumulativa da atenuação por chuvas para os 6 enlaces convergentes de Brasília. $\quad 68$

Tabela 15 Distribuição cumulativa da atenuação diferencial por chuvas para os pares de enlaces convergentes de Brasília que o consideram INCRA-BSA. 68

Tabela 16 Média dos erros (Recomendação P.311).

Tabela 17 Desvio padrão dos erros (Recomendação P.311). 71

Tabela 18 Valor RMS dos erros (Recomendação P.311). 72

Tabela 19 Média dos erros (Erro relativo). $\quad 74$

Tabela 20 Desvio padrão dos erros (Erro relativo). 75

$\begin{array}{ll}\text { Tabela } 21 \text { Valor RMS dos erros (Erro relativo). } & 75\end{array}$ 
Tabela 22 Valores das constantes de ajuste para o fator $r_{p}$.

Tabela 23 Média dos erros (Erro relativo), incluindo o modelo Pérez-Mello. 84

Tabela 24 Desvio padrão dos erros (Erro relativo), incluindo o modelo PérezMello.

Tabela 25 Valor RMS dos erros (Erro relativo), incluindo o modelo Pérez-Mello.

Tabela 26 Valores das constantes de ajuste para a atenuação diferencial por chuvas (Modelo 1). 99

Tabela 27 Valores das constantes de ajuste para o parâmetro $\eta$. 104

Tabela 28 Valores das constantes de ajuste para o parâmetro $\beta$.

Tabela 29 Média, desvio padrão e valor RMS do erro relativo para os modelos $1 \mathrm{e}$ 2.

Tabela 30 Duração média de eventos de chuva para o enlace CENESP15-RIS. 118

Tabela 31 Valores das constantes de ajuste para o parâmetro $\eta$.

Tabela 32 Valores das constantes de ajuste para o parâmetro $\beta$.

Tabela 33 Média, desvio padrão e valor RMS dos erros (Recomendação P.311-10 e Erro Relativo), para o modelo DEC-PM.

Tabela 34 Valores das constantes de ajuste para $\eta$ (Modelo DEC-PM Modificado).

Tabela 35 Valores das constantes de ajuste para $\beta$ (Modelo DEC-PM Modificado).

Tabela 36 Média, desvio padrão e valor RMS dos erros (Recomendação P.311-10) para os modelos DEC-PM e DEC-PM Modificado.

Tabela 37 Valores dos coeficientes do polinômio de ajuste.

Tabela 38 Valores das constantes dos coeficientes $b_{i}$.

Tabela 39 Média, desvio padrão e valor RMS dos erros (Recomendação P.311-10 e Erro Relativo), para o modelo DMEC-PM.

Tabela 40 Valores dos coeficientes $b_{i}$ (Modelo DMEC-PM modificado).

Tabela 41 Média, desvio padrão e valor RMS dos erros (Erro Relativo) para os modelos DMEC-PM e DMEC-PM Modificado.

Tabela 42 Comparação do raio da célula no sistema LMDS $\backslash$ LMCS, para os modelos de ITU-R e Pérez-Mello.

Tabela 43 Características dos enlaces da base de dados de ITU-R de atenuação por 
chuvas.

Tabela 44 Características dos pares de enlaces convergentes de São Paulo. 167

Tabela 45 Características dos pares de enlaces convergentes de Brasília. $\quad 168$

Tabela 46 Dados para o modelamento da duração de eventos de chuva no Brasil (modelos DEC-PM e DEC-PM Modificado). 